\title{
Developing Therapies that Prevent Progenitor Cell Migration for Preventing the Development and Further Growth of Endometriotic Lesions- Future Prospects-A Short Communication
}

ISSN: 2640-9666

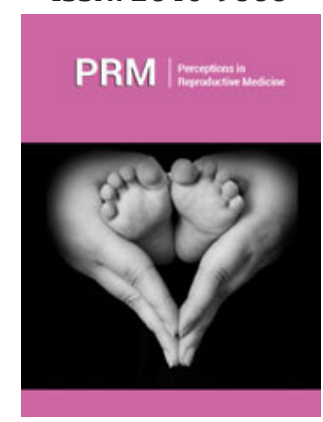

${ }^{*}$ Corresponding author: Kulvinder Kochar Kaur, Centre for Human Reproduction, India

Submission: 笽June 24, 2020

Published: 㠿July 10, 2020

Volume 3 - Issue 5

How to cite this article: Kulvinder Kochar Kaur, Gautam Allahbadia, Mandeep Singh. Developing Therapies that Prevent Progenitor Cell Migration for Preventing the Development and Further Growth of Endometriotic LesionsFuture Prospects-A Short Communication. Perception in Reproductive Medicine.3(5). PRM.000575.2020.

DOI: $10.31031 /$ PRM.2020.03.000575

Copyright@ Kulvinder Kochar Kaur, This article is distributed under the terms of the Creative Commons Attribution 4.0 International License, which permits unrestricted use and redistribution provided that the original author and source are credited.

\author{
Kulvinder Kochar Kaur*1, Gautam Allahbadia ${ }^{1}$ and Mandeep Singh ${ }^{2}$ \\ ${ }^{1}$ Centre for Human Reproduction, India \\ ${ }^{2}$ Department of Neurologist, India
}

\section{Short communication}

Endometriosis represents a frequent as well as benign gynecological disease that is defined as the presence of endometrial tissue (glands as well as stroma) in sites external to the normal endometrium as well as rest of the parts of uterus [1]. About $10 \%$ of women of childbearing age have endometriosis with the rate escalating in recent years. There is an immediate need for getting efficacious therapies. Earlier we had reviewed on the etiopathogenesis ,management of Endometriosis as well as Adenomyosis in infertile patients ,effect on oocytes along with Endometriosis in adolescents and effectively managing both pain as well as infertility along with preventing risk of malignancy in endometriosis but still no permanent solution is coming hence the endeavor to keep looking for newer avenues [2-8].

It is known that Endometriosis is an estrogen associated disease ,along with neovascularization having a crucial part in its establishment, propagation as well as recurrence .It has been shown that via mobilization as well as recruitment of endothelial progenitor cells (EPC's) estrogen has significant role in neovascularization $[9,10]$. Further estrogen can induce the endometriotic lesions escalating [11,12]; (Figure $1 \& 2$ ), with some recent studies illustrating that therapy with estrogen facilitates vasculogenesis in endometriotic lesions via enhancing amounts of EPC's [13]. Chemokine receptor 4(CXCR4) is a key manipulator regarding homing of EPC's [14], while Li et al. [13], demonstrated that estrogen escalates the recruitment of EPC's to the areas of damage through the stromal cell derived factor(SDF1)CXCR4 pathways in myocardial infarction(MI) [11]. Certain studies have illustrated that estrogen increases the SDF1/CXCR12 synthesis in human Endometrial stromal cells (HESCs) in a time as well as dose-based fashion [11]. Nevertheless, the part as well as mode of SDF1/ CXCR12 in endometriosis is not understood.

CK2 represents a serine/threonine kinase made up of 2 catalytic ( $\alpha$ or $\alpha^{\prime}$ )subunits as well as 2 regulatory ( $\beta$ ) subunits, that assemble in the form of a tetramer.CK2 can phosphorylate substrates to control a lot of biological events [15]. Earlier Feng et al. [16], showed that CK2 controls angiogenesis of endometriotic lesions along with a protein kinase CK2 inhibitor inhibits the development of new blood vessel's as well as growth of endometriotic lesions. Still it was not clear what is the insight in CK2 controls of EPC's at the time of vasculogenesis in endometriotic lesions [17]. Endometriosis has been known to be crucially based on actively going angiogenesis and thus new blood vessels represent a mark of the lesions. Trying to get methods of blocking this angiogenesis as well as vasculogenesis might help in innovative ways of tackling endometriosis. Bone marrow (BM)-derived endothelial cells aid in vasculogenesis, that includes the ones for endometriosis as well as uterus at the time of pregnancy [18]. In view of their part in endometriosis lesions as well as recruitment during active disease, these endothelial progenitor cells are good targets for therapeutic intervention. 


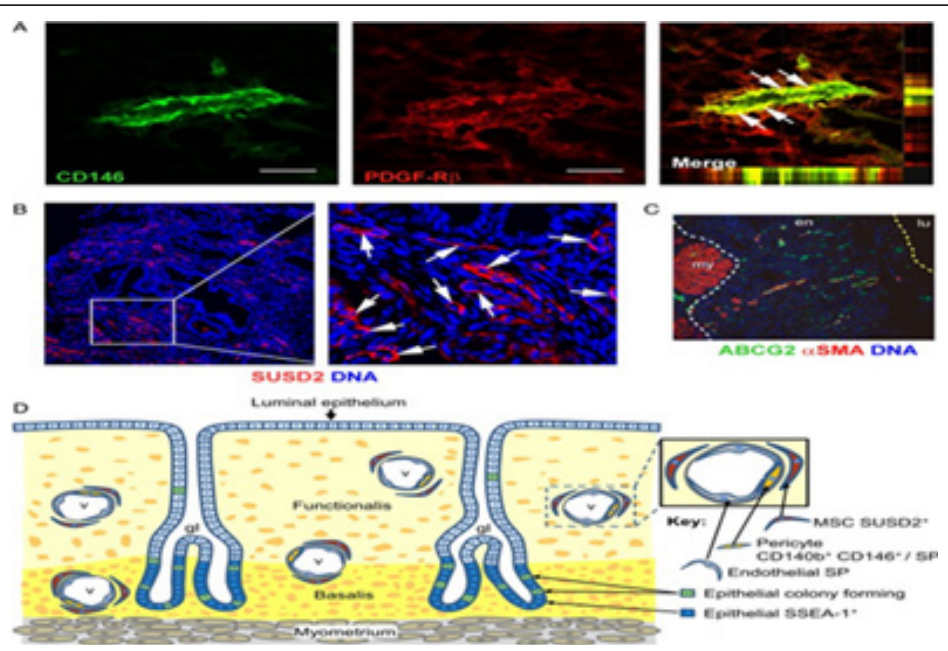

Figure 1: Courtesy ref no-12-Localization of human endometrial mesenchymal stem cells. (A-C) Immunofluorescence images of human endometrium showing perivascular identity of human eMSCs.

a. Co-localization (white arrows) of CD146 and platelet-derived growth factor receptor beta (PDGF-R $\beta$ ) in pericytes of venules and possibly capillaries in the functionalism stroma. The $\mathrm{x} / \mathrm{z}$ and $\mathrm{y} / \mathrm{z}$ planes are shown on the far right and underneath the merged images demonstrating co-localization of the two surface markers.

b. Perivascular SUSD2 expression (white arrows).

c. ATP-binding cassette, subfamily G member 2 (ABCG2) and aSMA co-staining showing perivascular and endothelial identity of SP cells. The white dotted lines indicate the junction between the endometrium (en) and myometrium (my) and yellow dotted line indicates the luminal surface (lu) of the uterine epithelium. (D) Schematic showing location of stem/progenitor cells identified in the human endometrium.

Epithelial progenitor cells are postulated to be a subpopulation of cells located in the base of the glands in the basalis, identified by SSEA-1. Sushi domain containing-2+ (SUSD2+) eMSCs are perivascular cells. eMSC co-expressing CD146 and PDGFR $\beta / C D 140 \mathrm{~b}$ are most likely pericytes, as they are located adjacent to endothelial cells in vessels (v) in both the basalis and the functionalism. SP cells are a heterogeneous population comprising CD31+ endothelial cells and $\mathrm{CD} 140 \mathrm{~b}+\mathrm{CD} 146+$ pericytes. Scale bar in $(\mathrm{A})=50 \mu \mathrm{m}$. (A) Reprinted with permissions from Schwab and Gargett [12]. (C) Reprinted with permissions from Masuda et al. (2010). (D) Adapted from Gurung et al. (2015).

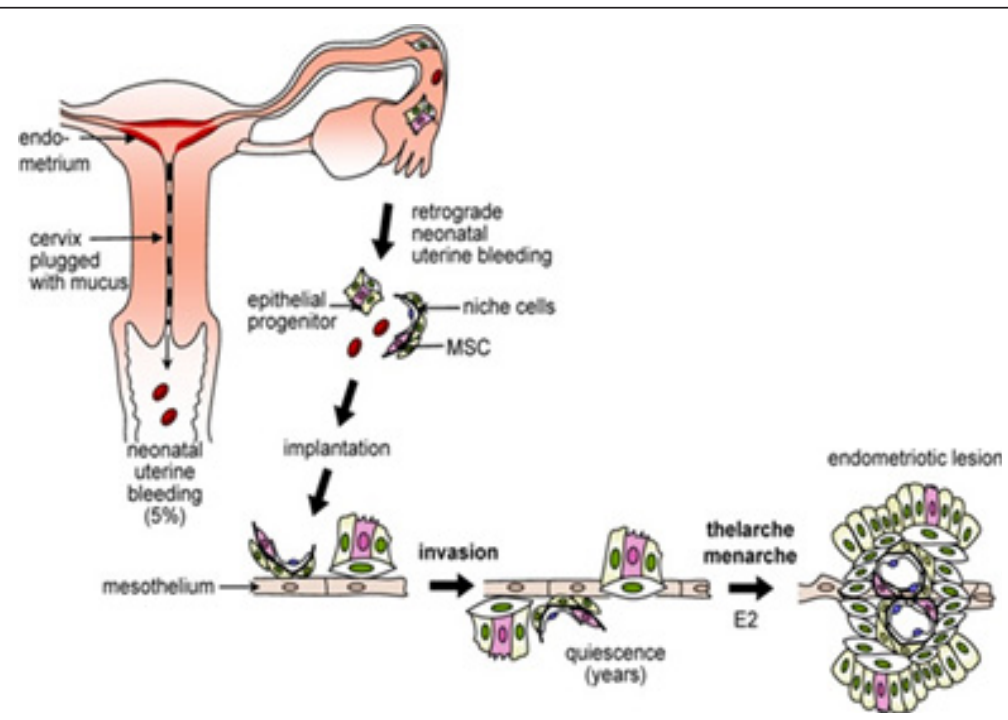

Figure 1: Courtesy ref no- 12-Schematic describing the hypothesis that endometrial stem/progenitor cells shed in neonatal uterine bleeding may play a role in early onset endometriosis. Neonatal uterine bleeding occurs in $5 \%$ of neonates. It is hypothesized that retrograde neonatal bleeding occurs because thick mucus obstructs the long neonatal cervix. Fragments of shed endometrial tissue are postulated to contain an endometrial epithelial progenitor cell (pink) and a perivascular MSC (pink) together with niche cells. These rapidly adhere to the neonatal mesothelium, invade and/or become contiguous with the mesothelial lining where they remain quiescent for $\square 10$ years. Rising estrogen (E2) levels associated with thelarche and menarche reactivate the stem/progenitor cells to initiate growth of endometriosis lesions on the surface of or below the peritoneal mesothelium, resulting in early onset endometriosis. Reprinted with permissions from Gargett et al. (2014). 
Both in endometrial as well as decidual physiology as well as pathology like endometriosis bone marrow (BM)-derived cells have a proven part. Stem cells via BM engraft normal endomerium and help in this endometrium repair following injury. BM-derived stem cell become decidual cells along with support pregnancy playing a necessary role [19]. Thus these BM-derived stem cells get transported within the circulation along with getting recruited to along with engrafted in endomerium is a part of normal physiology, CXCL12 represent a strong chemoattractant which results in migration as well as engraftment of BM cells in normal endomerium as well as Endometriosis [19]. Taylor's group earlier demonstrated that endometriosis synthesis large amounts of CXCL12 (also called stromal derived factor 1 [SDF1] [20]. Estrogen activates the synthesis as well as liberation of CXCL12. BM cells which get moved to e endometriosis by the attraction of CXCL12 are a subpopulation of endothelial progenitor cells. These endothelial progenitor cells are circulating cells which stick to the endomerium at the areas of hypoxia or ischemia and aid in new vessel development. These stem cells getting incorporated is key for blood vessels growth as well as progression of endometriosis.

The control of CXCL12 expression by the protein kinase CK2 has been found by Zhao et al. [21]. The same group earlier demonstrated that CK2 controls angiogenesis within these Endometriosis lesions. Blockade of this enzyme avoids development of new blood vessels as well as growth of Endometriosis. This ubiquitous as well as constitutively active serine threonine kinase has been shown to be controlled via estrogen, whose activity thus escalates CXCL12. This CXCL12 attracts endothelial progenitor cells, by a mode by which these cells get homed to endometriosis lesions. By blocking this path at any junction blocks endothelial cell migration, depletion of estradiol(E2). On blocking CK2 action with its potent as well as selective inhibitor CX4945, or with usage of CXCL12 receptor antagonist AMD3100 all inhibit this signaling path. In the same way Taylor showed in animal models of endometriosis recently regarding AMD3100 inhibits stem cell migration to endometriosis and thus results in regression of these lesions [22].

\section{Conclusion}

Will therapy with agents that block CK2 have the capacity as treatment of endometriosis? CK2 represents a pleiotropic protein kinase, that controls lot of growth as well as survival paths in normal cells as well as disease. Though CK2 inhibitors CX4945 has been formed for treatment of cancers, with its nonspecific effect on practically all tissues, risk of off target actions is high, and thus makes it unlikely to be used for endometriosis therapy .But this gives a lot of lessons regarding blockade of signaling path that ends in endothelial cell engraftment of endometriosis. Inhibition of CXCL12. Action by using AMD3100 holds promise and has realistic new way of Endometriosis therapy as shown in murine models of Endometriosis [23]. More significant, we get insight that estrogen depriving controls CK2, CXCL12 as well as endothelial cell migration.
Progestin therapy does not add this benefit. The therapies used currently for decreasing Estrogen are Gn RH agonists, aromatase inhibitors. Though this suddenly is not possibly help in future forming agents which block CK2 to be included as agents for Endometriosis therapy regimens we have got insight that standard therapies will inhibit endothelial cell engraftment as well as lesion expansion. Considering non hormonal therapy CXC R4 antagonist have>specificity as well as less toxic as compared to CK2 inhibitors, but action on pregnancy is not well known. Decrease of estrogen will also block cell engraftment. Thus, E2 deprivation remains gold standard for Endometriosis therapy till now.

\section{References}

1. Giudice LC (2010) Endometriosis. N Engl J Med 362: 2389-2398.

2. Kulvinder Kochar K, Allahbadia GN, Singh M (2017) Current controversies and future challenges in endometriosis therapy. EC Gynaecology 1: 9-13.

3. Kulvinder Kochar K, Allahbadia GN, Singh M (2016) An update on pathophysiology and medical management of endometriosis. Advances in Repr Sci 4(2): 53-73.

4. Kulvinder Kochar K, Allahbadia GN, Singh M (2017) Meeting the challenges of endometriosis associated pain-newer options for future and research directions. BAOJ Bioinfo 1: 9.

5. Kulvinder Kochar K, Allahbadia GN, Singh M (2019) An update on diagnosis and management of adolescent endometriosis -A short communication. Acta Scientific Paediatrics 2(5): 48-50.

6. Kulvinder Kochar K, Allahbadia GN, Singh M (2019) Dissecting the reduced ovarian reserve seen with ovarian enometriomas with that caused by surgical excision of ovarian enometriomas-what comes first. Surg Med Open Acc J 2(2).

7. Kulvinder Kochar Kaur, Allahbadia GN, Singh M (2020) How can we optimize the granulose cell function of oo $\urcorner$ cytes from an endometriosis patient to im $\urcorner$ prove the reproductive outcomes-A short communication? Perception in Reproductive Medicine 3(4).

8. Kulvinder Kochar Kaur, Allahbadia GN, Singh M (2020) An update on the various theories of the origin of uterine adenomyosis-A systematic review. Archives of Urology 3(1): 31-45.

9. Streblow K, Werner N, Berweiler J, Link A, Dirnagl U, et al. (2003) Estrogen increases bone marrow derived endothelial progenitor cells production and diminishes neointima formation. Circulation 107(24): 3059-3065.

10. Auth RJ, Nenicu A, Nickels RM, Menger MD, Laschke MW (2016) Estrogen stimulates homing of endothelial progenitor cells to endometriotic lesions. Am J Pathol 186: 2129-2142.

11. Tsutsumi A, Okada H, Nakamoto N, Oka Moto R, Yasuda K, et al. (2011) Estrogen induces stromal cell derived factor-1(SDF1/CXCR12) production in human endometrial stromal cell: A possible role of endometrial epithelial cells. Fertil Steril 95: 444-447.

12. Gargett CE, Schwab KE, Deane JA (2016) Endometrial stem/progenitor cells: The first ten years. Human Reprod Update 22(2): 137-163.

13. Li H, Liu J, YeX, Zhang X, Wang Z, et al. (2013) 17ß-Estradiol enhances the recruitment of bone marrow derived endothelial progenitor cells into infar cted myocardium by inducing CXCR4 expression. Int J Cardiol 162(2): 100-106.

14. Sainz J, Sata M (2007) CXCR4 a key modulator of vascular progenitor cells. Arterioscler Thromb Vasc Biol 27: 263-265.

15. Shehata M, Schnabi S, Demirtas D, Hilgarth M, Hilgarth M, et al. (2010) Reconstitution of PTEN activity by CK inhibitors and interference with the PIK3/Akt cascade counteract the antiapoptotic effect of human 
stromal cell in chronic lymphocytic leukaemia. Blood 116: 2513-2521.

16. Feng D, Welker S, Korbel C, Auth RJ, Menger MD, et al. (2012) Protein kinase CK2 is a regulator of angiogenesis of Endometriotic lesions. Angiogenesis 15(2): 243-252.

17. Montenarh M (2014) Protein kinase CK2 and angiogenesis. Adv Clin Exp Med 23(2): 153-158.

18. Tal R, Dong D, Shaikh S, Manallapalli R, Taylor HS (2019) Bone marrowderived endothelial progenitor cells contribute to vasculogenesis of pregnant mouse uterus. Biol Reprod 100(5): 1228-1237.

19. Tal R, Shaikh S, Pallavi D, Tal A, Giraldez LF, et al. (2019) Adult bone marrow progenitors become decidual cells and contribute to embryo implantation and pregnancy. PLoS Biol 17(9): e3000421.
20. Li F, Alderman MH, Tal A, Manallapalli R, Coolidge A, et al. (2018) Haematogenous dissemination of mesenchymal stem cells from Endometriosis. Stem Cells 36(6): 881-890.

21. Zhao RM, Feng D, Zhuanf G, Liu Y, Chi S, et al. (2020) Protein kinase CK2 participates in estrogen mediated endothelial cell homing to Endometriosis lesions through stromal cells in an SDF1- CXC R4 dependent manner. Fertil Steril 113(5): 1067-1079.

22. Plochino N, Manallapalli R, Shaikh S, Habata S, Tal A, et al. (2020) CXC R4 or CXC R7 antagonists treat endometriosis by reducing bone marrow cell trafficking. J Cell Mol Med 24: 2464-2474.

23. Taylor HS (2020) Bone marrow in the pathophysiology of Endometriosis. Fertil Steril 113: 942-942.

For possible submissions Click below: 